sciendo

Journal of Research in Physics

Vol. 39, No. 1, $65-74(2018)$

doi:10.2478/jrp-2018-0006

\title{
Calculation of the optical transmittance in cadmium oxide by considering oxygen plasma
}

\author{
M. A. Grado-Caffaro and M. Grado-Caffaro \\ C/ Julio Palacios 11, 9-B, 28029-Madrid, Spain \\ E-mail: ma.grado-caffaro@sapienzastudies.com
}

Received: February 5, 2018

\begin{abstract}
By considering the partial pressure of oxygen in the deposition process of cadmium oxide, we calculate the maximum transmittance of cadmium oxide in the visible range, which is achieved at the so-called optimum partial pressure of oxygen, this value of pressure being a key figure of merit. Our formulation agrees with experimental measurements and is consistent with the so-called plasma-optical effect. At this point, an exhaustive evaluation of previous experimental data is performed by doing a detailed comparison among the results from different authors and by performing numerical estimations (including estimations from experimental data) of relevant physical quantities. In fact, a comprehensive discussion relative to comparison between experimental measurements and our theoretical results is presented.
\end{abstract}

Key words: cadmium oxide, optical transmittance, visible range, partial pressure of oxygen, optimum pressure.

\section{Introduction}

The formation of certain molecules from metals in the presence of oxygen plasma is a relevant issue with a number of implications of obvious importance in Optical Physics, Molecular Physics, and Plasma Physics. In this context, we can mention vacuum evaporation of a metal in the presence of oxygen plasma to form a conducting or semiconducting oxide. As a relevant example, one may regard the role of cadmium oxide (a very transparent II-VI, n-type, semiconductor oxide) which is really important in the optoelectronics area. Among a number of applications in this area, $\mathrm{CdO}$ serves as antireflection coating for solar cells [1-5] although, however, is not widely used (undoped) due to being really hazardous [6,7]. Nevertheless, if this oxide is doped, for example, by indium, then its usefulness as coating in solar cells becomes reasonable. Cadmium oxide has been investigated experimentally in refs. $[5,8-21]$ in relation to electrical, optical, and thermodynamical properties as well as theoretically in refs.[22-28]. Unfortunately, questionable work on the subject can be found in refs. [29-32]. Certain issues related to this interesting material remain to 
be investigated either experimentally or theoretically. We refer, in particular, to questions concerning the influence of the partial pressure of oxygen in the deposition process of $\mathrm{CdO}$ upon its electrical and optical properties. In this context, we should mention $\mathrm{CdO}$ thin films prepared by reactive evaporation [5,8-12].

In relation to the optical properties of cadmium oxide, there is a crucial issue, namely, the transmittance in the visible range. It has been found that the optical properties of $\mathrm{CdO}$ exhibit sharply decreasing transmittance at higher photon energies so this means that the oxide in question behaves mainly as a direct-gap semiconductor (see, for instance, refs. [5,810]). As a matter of fact, the transmittance is large in the visible range and relies upon the partial pressure of oxygen during the deposition process [5,8-12]. The value of this pressure at which the transmittance reaches its maximum value is called optimum pressure. In this respect, it is known that the decrease in transmittance at values of the partial pressure of oxygen larger than the above mentioned optimal value arises from increased light scattering [5,8-10]. On the other hand, it is also known that $\mathrm{CdO}$ films are non-stoichiometric (leading to decreasing transmittance) when the oxygen partial pressure is smaller than the optimum pressure [5,8-10]. Really, the optimum partial pressure of oxygen is a key figure of merit which, in association with other relevant parameters, has been measured experimentally by some authors as in refs.[5,8-12]. In fact, a value of $1 \cdot 10^{-3}$ Torr was measured [5]. Surprisingly, a value of $0.04 \mathrm{~Pa}$ (approximately $3 \cdot 10^{-3}$ Torr), roughly 3 times the former value [5], was given in refs. [11,12].

In the present paper, we will calculate the averaged transmittance of $\mathrm{CdO}$ in the visible region. At this point, we must remark that we regard the above transmittance as a function of the partial pressure of oxygen so, as we have said before, the maximum value of the transmittance is attained at the optimum partial pressure of oxygen. It should be pointed out that the above mentioned averaged transmittance means the maximal transmittance averaged over a set of relevant parameters as we will see later. As a matter of fact, we will arrive at an analytical expression and, finally, by using (in part) values of relevant parameters measured experimentally, we shall give an approximate value for the (average) transmittance which is in good agreement with experimental observations. We will start from reasonable considerations on optical absorption and reflectance in the visible range and apply the well known Tauc's formula for the coefficient of optical absorption relative to direct-gap semiconductors [33-35]. This formula assumes reasonably parabolic conduction bands so non-parabolic effects can be neglected here. The paper is organized as follows: section 2 is devoted to the calculation of the maximal transmittance of $\mathrm{CdO}$ in the visible region; section 3 describes the evaluation of experimental data obtained by some authors; section 4 represents the discussion of results; concluding remarks are given in section 5 .

\section{Quantitative description}

Considering $\mathrm{CdO}$ thin films, we start from the following expression:

$$
T+R+A=1
$$

where $T, R$ and $A(A<<1)$ denote transmittance, reflectance, and absorbance, respectively. In particular, we have that $A \approx \alpha t$ (if $A<<1$ ) where $\alpha$ and $t$ are the coefficient of optical absorption and film thickness, respectively. 
On the other hand, the well-known Tauc's formula gives the direct transition permitted between the parabolic bands as follows [33-35]:

$$
\alpha(\omega)=\frac{k \sqrt{\hbar \omega-E_{g}}}{\hbar \omega}
$$

where $E_{g}$ is the optical band-gap energy, $k$ is a non-negative real constant which is viewed as an edge-width parameter associated with energy bands and effective masses, and, of course, $\hbar \omega$ is the photon energy.

Since $\mathrm{CdO}$ is a quasi-antireflecting material, then one has that $R \approx 0$ which, together with formulae (1), (2) and that $A \approx \alpha t$, yield:

$$
T\left(\omega, E_{g}, t\right) \approx 1-\frac{k t \sqrt{\hbar \omega-E_{g}}}{\hbar \omega}
$$

Relationship (3) refers to the maximum transmittance which means that $E_{g}$ (which depends on the partial pressure of oxygen) corresponds to the optimum pressure and varies only by virtue of the so-called Burstein-Moss effect. In addition, from experimental considerations [5,8-10], the film thickness $t$ is conceived as variable; in fact, it was determined by employing spectrophotometric techniques and its values changed between $2.0 \mu \mathrm{m}$ and $2.5 \mu \mathrm{m}[5,8-10]$.

Consequently, we may consider the average transmittance of $\mathrm{CdO}$ in the visible region as follows:

$$
\langle T\rangle=\frac{1}{\left(\omega_{2}-\omega_{1}\right)\left(E_{g 2}-E_{g 1}\right)\left(t_{2}-t_{1}\right)} \iiint_{D} T\left(\omega, E_{g}, t\right) d \omega d E_{g} d t
$$

where $D=\left[\omega_{1}, \omega_{2}\right] \times\left[E_{g 1}, E_{g 2}\right] \times\left[t_{1}, t_{2}\right]$. As we have remarked in section 1 , notice that the above averaged transmittance becomes maximal with respect to the partial pressure of oxygen which, from now on, will be denoted by $P$. In fact, the aforementioned maximum value is achieved at the optimum partial pressure of oxygen which, from now on, will be designated by $P_{0}$ (see section 1 ).

Replacing (3) into (4), we have easily:

$$
\langle T\rangle \approx 1-\frac{k}{\hbar\left(\omega_{2}-\omega_{1}\right)\left(E_{g 2}-E_{g 1}\right)\left(t_{2}-t_{1}\right)} \int_{t_{1}}^{t_{2}} t d t \int_{\omega_{1}}^{\omega_{2}} \frac{d \omega}{\omega} \int_{E_{g 1}}^{E_{g 2}} \sqrt{\hbar \omega-E_{g}} d E_{g}
$$

Formula (5) becomes:

$$
\langle T\rangle \approx 1+\frac{2 k\left(t_{1}+t_{2}\right)}{3 \hbar\left(\omega_{2}-\omega_{1}\right)\left(E_{g 2}-E_{g 1}\right)} \int_{\omega_{1}}^{\omega_{2}}\left[\left(\hbar \omega-E_{g 2}\right)^{3 / 2}-\left(\hbar \omega-E_{g 1}\right)^{3 / 2}\right] \frac{d \omega}{\omega}
$$

Applying eq. (2) and known (averaged) values of the involved quantities, we have found that $k \approx 5.2 \cdot 10^{5} \mathrm{~cm}^{-1}(\mathrm{eV})^{1 / 2}$, which agrees well with the corresponding value extracted from fig. 3 in ref. [5]. By using the above value for $k$ and $t_{1}=2 \mu \mathrm{m}, t_{2}=2.5 \mu \mathrm{m}$, changing the variables from $\omega$ to a new variable, say, $x$ such that $\hbar \omega-E_{g 1,2}=x$ in the integral of eq. (6) and taking $\hbar \omega_{1} \approx E_{g 1} \approx 2.3 \mathrm{eV}, \hbar \omega_{2} \approx 3.26 \mathrm{eV}$ (the visible region is between $1.65 \mathrm{eV}$ and $3.26 \mathrm{eV}$ ), after simple computation, it follows that $\langle T\rangle \approx 0.87$ which is in good agreement with experimental data [5,8-12] (see table 1 and fig. 1). The key 
optical parameters of $\mathrm{CdO}$ at the optimum partial pressure of oxygen are given in Table 1; however, $k$ does not depend on $P$. Fig. 1 is a schematic diagram related to the formation of $\mathrm{CdO}$ and the influence of the partial pressure of oxygen during the deposition process of the material in question on the optical properties of $\mathrm{CdO}$ in the visible region.

Table 1: Key optical parameters of $\mathrm{CdO}$ at $P_{0}$.

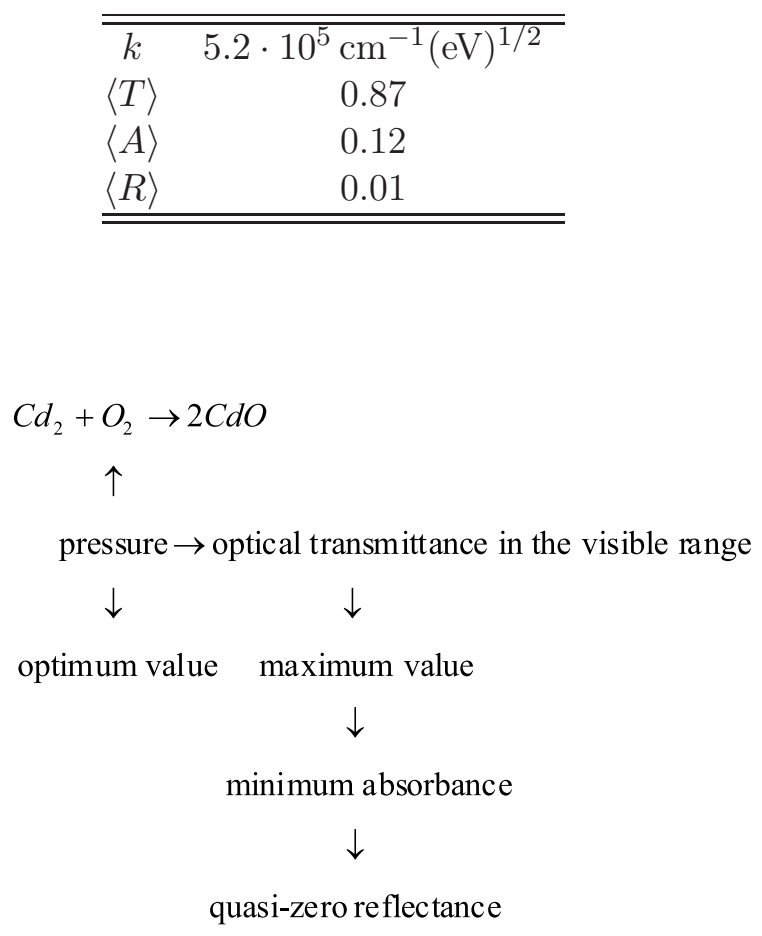

Figure 1: Schematic diagram relative to the formation of $\mathrm{CdO}$ and its optical properties.

\section{Evaluation of experimental data}

$\mathrm{CdO}$ thin-film deposition has been made by means of various techniques [5,8-14]. Within this context, it is very important to get more reproducible thin films of high quality. This is achievable by employing, for instance, activated reactive evaporation [5,8-10], where one may appreciate the evident influence of the partial pressure of oxygen on the physics of $\mathrm{CdO}$ thin films during the deposition process of the aforementioned oxide (see fig. 1). In fact, the films were prepared by using a Hind Hi Vac coating unit by evaporating elemental cadmium in the presence of oxygen plasma in vacuum. First, the chamber was evacuated to a pressure smaller than $2 \cdot 10^{-5}$ Torr. Glass substrates were maintained at $200{ }^{\circ} \mathrm{C}$ [5]. The deposition rate was fixed at $1 \mathrm{~nm} / \mathrm{s}$ and the glow power was kept at $2 \mathrm{~W}$ [5]. On the other hand, the partial pressure of oxygen was changed between $10^{-2}$ Torr and $10^{-4}$ Torr. The films presented cubic structure with $\left(\begin{array}{lll}1 & 1 & 1\end{array}\right)$ preferred orientation [5].

In refs. [11,12] the experimental results have been obtained by using reactive evaporation and are in qualitative agreement with refs. [5,8-10]. At this point, we should point out that the experimental technique corresponding to refs. [11,12] is essentially the same as in refs. [5,8-10] and ref. [20]. Ref. [20] deals with the vacuum evaporation method 
on a glass substrate at room temperature. Structural, electrical, and optical properties of $\mathrm{CdO}$ thin films were determined at several annealing temperatures [20]; in particular, the coefficient of optical absorption was measured at the fundamental absorption region [20]. Optical transmittance in the visible region was measured yielding results between $70 \%$ and $92 \%$ [11,12] which agrees with experimental data in ref. [5] (see table 2).

Table 2: Optical transmittance of $\mathrm{CdO}$ in the visible range (from ref. [5]).

\begin{tabular}{lc}
\hline$\overline{P(\text { Torr })}$ & $T$ \\
\hline $2 \cdot 10^{-4}$ & 0.85 \\
$1 \cdot 10^{-3}$ & 0.90 \\
$5 \cdot 10^{-3}$ & 0.80 \\
$1 \cdot 10^{-2}$ & 0.75 \\
\hline \hline
\end{tabular}

We wish to emphasize that $E_{g}$ depends on $P$ so we rewrite eq. (2) as follows:

$$
\alpha(\omega, P)=\frac{k \sqrt{\hbar \omega-E_{g}(P)}}{\hbar \omega}
$$

Looking at eq. (7), we may say that $P$ acts as a parameter. Eq. (7) defines a family of plane curves so, for each value of $P$ within the range indicated in Table 2 [5,8-12], one has a particular curve. On fig. 3 of ref. [5] a family of curves (for the values of $P$ quoted in table 2 , except the third value) is plotted taking the photon energy $\hbar \omega$ as abscissa and $(\alpha \hbar \omega)^{2}$ as ordinate. It should be mentioned that an extrapolating computation for these curves was done in [23] so a set of curves passing by the point $\left(2 \mathrm{eV}, 0.01(\mathrm{eV} / \mathrm{cm})^{2}\right)$ was found [23]. However, the reader can easily appreciate that the above extrapolation is not consistent with formula (7) because the condition $\hbar \omega \geq E_{g}(P)$ is not satisfied. Under this condition, let us consider the interesting case in which $\hbar \omega \approx E_{g}\left(P_{0}\right)$, that is, when the band-gap energy approximately equals the photon energy corresponding to the frequency of the visible radiation at which the transmittance has its maximum. Consequently, it is clear that this case corresponds to the minimal coefficient of absorption so that one may replace the denominator of $(7)$, namely $\hbar \omega$, by $E_{g}\left(P_{0}\right)$ so formula (7) becomes an expression for the minimum absorption coefficient (see formula (1)) as follows:

$$
\alpha_{\min } \approx k \sqrt{\frac{\hbar \omega_{0}}{E_{g}^{2}\left(P_{0}\right)}-\frac{1}{E_{g}\left(P_{0}\right)}}
$$

where $\omega_{0}$ is the angular frequency of the visible radiation at which the transmittance reaches its maximum value.

On the other hand, it is clear that relationship (8) may be employed to determine $E_{g}\left(P_{0}\right)$ by knowing $\alpha_{\min }[5]$ so, from formula (8) it follows explicitly:

$$
E_{g}\left(P_{0}\right) \approx \hbar \omega_{0}\left(1-\frac{\hbar \omega_{0} \alpha_{\min }^{2}}{k^{2}}\right)
$$


However, we must remark that $E_{g}\left(P_{0}\right)$ is not a fixed value due to the Burstein-Moss shift which, within the quasi-free electron theory, reads:

$$
\Delta E_{g}\left(P_{0}\right)=\frac{\hbar^{2}}{2 m_{r}^{*}}\left[3 \pi^{2} n\left(P_{0}\right)\right]^{3 / 2}
$$

where $m_{r}^{*}$ and $n\left(P_{0}\right)$ denote electron-hole reduced effective mass and electron concentration (CdO is n-type), respectively. It should be noted that the electron concentration depends on $P$ (see, for instance, refs. [5,8-10]). In fact, from refs. [5,8-10] and our numerical estimations, we have obtained table 3 where approximate values of $n(P)$, corresponding to the same values of $P$ relative to table 2 , are given. Furthermore, we have determined $n_{\text {max }} \approx 12.7 \cdot 10^{19} \mathrm{~cm}^{-3}$ at $P \approx 8 \cdot 10^{-4}$ Torr, which is relatively close to $P_{0}$. It seems that the value of $P$ (for $n_{\max }$ ) extracted from fig. 2 in ref. [5], is slightly larger than our above mentioned value of $P$, so we may conclude that the maximal electron spatial density is reached at (roughly) the optimum partial pressure of oxygen [5]. On the other hand, we note that, for the first three values of pressure in table 3, the electron concentration is practically the same except for the second value which, however, corresponds to a value of $n$ slightly larger than the first and third values. Also, one can see that for the fourth value of $P$, the electron concentration is much smaller than the other values of $n$.

Table 3: Electron concentration for some relevant values of $P$.

\begin{tabular}{lc}
\hline$\overline{P(\text { Torr })}$ & $n\left(10^{19} \mathrm{~cm}^{-3}\right)$ \\
\hline $2 \cdot 10^{-4}$ & 11.2 \\
$1 \cdot 10^{-3}$ & 12.5 \\
$5 \cdot 10^{-3}$ & 11.3 \\
$1 \cdot 10^{-2}$ & 2.0 \\
\hline \hline
\end{tabular}

We have that $m_{r}^{*}=m_{e}^{*} m_{h}^{*} /\left(m_{e}^{*}+m_{h}^{*}\right)$ where $m_{e}^{*}$ and $m_{h}^{*}$ are the effective masses of electrons and holes, respectively. Since in $\mathrm{CdO}$ one has that $m_{h}^{*}>>m_{e}^{*}$, then, by the above formula, we have that $m_{r}^{*} \approx m_{e}^{*}$ which, inserted into (10) together with $\left(3 \pi^{2}\right)^{2 / 3} \approx$ $\left(\pi^{3}\right)^{2 / 3}=\pi^{2}$ leads to:

$$
\Delta E_{g}\left(P_{0}\right) \approx \frac{\pi^{2} \hbar^{2}\left[n\left(P_{0}\right)\right]^{2 / 3}}{2 m_{e}^{*}}
$$

The aforementioned quantity $E_{g}\left(P_{0}\right)$ is, in reality, $E_{g}^{(0)}\left(P_{0}\right)+\Delta E_{g}\left(P_{0}\right)$ where the second summand is, of course, given by relation (11) and the first one represents band-gap energy without taking into account the Burstein-Moss shift.

\section{Discussion of results}

Starting from formulas (2) and (3), we have obtained an approximate value of $\langle T\rangle$ (see section 2). We recall that this value is maximal and it is attained at the optimum value of $P$. This is in the context of table 2. In this respect, in order to generalize, one may think 
on averaged transmittance by considering $N$ points from fig. 2. in ref. [5], Therefore, we can write, as average transmittance:

$$
\langle T\rangle=\frac{\sum_{i=1}^{N} P_{i} T_{i}}{\sum_{i=1}^{N} P_{i}}
$$

For $N=4$, by using formula (12) and table 2 , one finds $\langle T\rangle \approx 77.6 \%$. On the other hand, if $N \rightarrow \infty$, by fixing $\omega$ and $t$ and taking into account that $E_{g}$ depends on $P$, then relationship (12) becomes, for a finite pressure range, say, $P_{1} \leq P \leq P_{2}$ :

$$
\langle T\rangle=\frac{1}{P_{2}-P_{1}} \int_{P_{1}}^{P_{2}} T(P) d P
$$

Substituting (3) into (13), by regarding that $\omega \geq \omega_{1}$ (see section 2), applying the Cauchy-Schwarz inequality, and considering a relatively small pressure range (for instance, an interval centered around $P_{0}$ ) so that the integral of $E_{g}(P)$ over the above range may be neglected, then the reader can verify the following estimation:

$$
\langle T\rangle<1+\frac{k t}{\omega_{1}} \sqrt{\frac{\omega_{2}}{\hbar}}
$$

The validity of centering the above mentioned pressure interval around $P_{0}$ should be a relevant fact given the important physical meaning of $P_{0}$. The reader can notice the usefulness of relationship (14) as an expression quasi-independent of both $P_{1}$ and $P_{2}$.

The electrical and optical properties of cadmium oxide acquire a notorious character in $\mathrm{CdO}$ nanowires and quantum dots (see fig. 2). On the other hand, it is well-known that both metallic and semiconductor nanostructures have gained relevance but still much work must be done on the subject in spite the important findings that have been achieved to date. As examples of these findings, we can mention refs. [36-45]. Fig. 2 is a schematic diagram relative to a $\mathrm{CdO}$ quantum dot. Assuming it as heavily doped (for example, indium heavily-doped), it is clear that the oxide in question becomes highly degenerate. In fact, the conduction electrons inside the dot behave as a very degenerate Fermi gas (possibly dilute). Actually, quantum dots based on II-VI semiconductor oxides are very relevant nanodevices which are attracting great attention. In particular, $\mathrm{CdO}$ nanoparticles (see fig. 2 and ref. [46]) exhibit significant optical properties; in this respect, we should wish to mention optical absorption, which was determined experimentally [46]. In addition, theoretical aspects related to the optical band-gap of the above mentioned nanoparticles (after the effective-mass approximation) were outlined in [46].

We have performed a comprehensive analysis on the transmittance (in the visible region) of $\mathrm{CdO}$ thin films from the theoretical point of view in combination with an exhaustive discussion relative to key experimental work developed by relevant investigators. We have studied carefully the work of authors whose experimental results agree well with our theoretical findings. Nevertheless, we must say that, speaking in general terms, experimental work in Condensed Matter Physics (as, also, in other branches of Physics) depends, sometimes in a crucial manner, upon a number of conditions. From this fact, apart from other issues, one may think the discrepancy among experimental observations as "natural" and the subject of the present paper is not an exception of the above mentioned considerations. At any rate, we can claim that experimental results of refs. [5,8-10] are reliable enough while refs. [11-15] are reliable to a certain extent. 


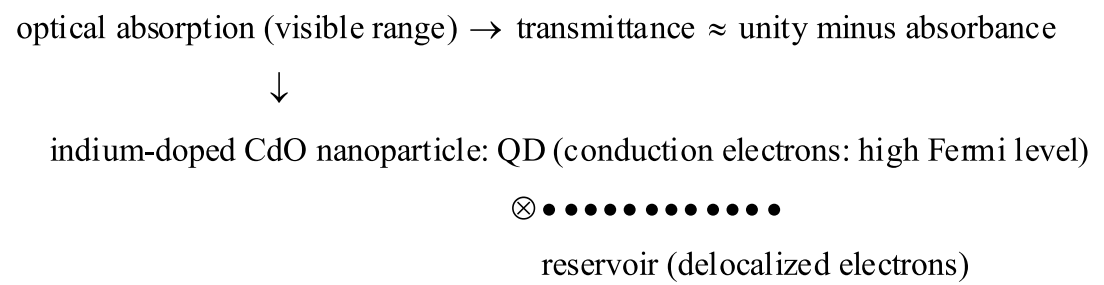

Figure 2: Optical behaviour of an indium-doped $\mathrm{CdO}$ quantum dot (QD) coupled to a reservoir.

Investigating the optical properties of transparent semiconducting oxides from the theoretical standpoint by using analytical tools, is a productive task. On the other hand, continuing with the experimental work in this field is also necessary to improve the experimental techniques. At any rate, elaborating solid theoretical models should be desired but, unfortunately, one must say that, by exploring the current literature, wrong theoretical schemes as well as misleading and clearly non-reliable experimental results can be found. Also, mixing non-transparent computer simulations with unreliable experimental tasks can be found in refs. [29-32] for example. Speaking of numerical simulations of refs. [29-32], we suspect that in a number of cases these simulations have been done by forcing the agreement of their results with experimental data which, on the other hand, may be false.

\section{Concluding remarks}

It is well-known that semiconductor oxides which are transparent in, at least, the visible region, are very attractive materials with a number of applications in Optoelectronics. In this context, one may also consider the behaviour of the above mentioned oxides in the infrared and ultraviolet ranges. Unfortunately, relevant theoretical problems regarding, for example, exciton-photon interaction in the oxides in question are missing in the current literature. Only inaccurate approaches on the aforementioned subject can be found, as in refs. $[47,48]$ for instance. In particular, $\mathrm{CdO}$ plays a significant role despite some problems that can be solved by doping (see section 1). In relation to experimental research in optical properties of cadmium oxide, one may claim that this research [5,8-20] can be viewed as reasonably satisfactory. But, at any rate, continuing experimental observations is necessary as well as theoretical-analytical work. On the other hand, let us recall that $\mathrm{CdO}$ offers special features in nanoelectronic devices (let us consider $\mathrm{CdO}$ quantum dots and nanowires) (see section 4 and ref. [46]).

\section{References}

[1] T. L. Chu, S. S. Chu, J. Electron. Mater 19, 1003 (1990).

[2] I. Shi, S. Jater, C. H. Champness, N. Liria, Solar Cells 7, 327 (1982).

[3] K. Lal Chopra, S. Ranjan Das, Thin film solar cells (Plenum Press, New York, 1983). 
[4] Y. Hames, S. E. San, Solar Energy 77, 291 (2004).

[5] C. Sravani, K. T. R. Reddy, P. J. Reddy, Mater. Lett. 15, 356 (1993).

[6] S. Calnan, A. N. Tiwari, Thin Solid Films 518, 1839 (2010).

[7] S. Calnan, Coatings 4,162 (2014).

[8] C. Sravani, K. T. R. Reddy, P. J. Reddy, Semicond. Sci. Technol. 6, 1036 (1991).

[9] C. Sravani, K. T. R. Reddy, O. M. Hussain, P. J. Reddy, J. Solar Energy Soc. India 6, 1 (1996).

[10] K. T. R. Reddy, C. Sravani, R. W. Miles, J. Cryst. Growth 184/185, 1031 (1998).

[11] F. C. Eze, Global J. Pure Appl. Sci. 9, 567 (2003).

[12] F. C. Eze, Mater. Chem. Phys. 89, 205 (2005).

[13] A. A. Dakhel, Curr. Appl. Phys. 11, 11 (2011).

[14] R. Rajammal, K. Rajaram, E. Savarimuthu, S. Arumugam, J. Nano-Electron. Phys. 3, 499 (2011).

[15] W. Dong, C. Zhu, Opt. Mater. 22, 227 (2003).

[16] M. Ristic, S. Popovic, S. Music, Mater. Lett. 58, 2494 (2004).

[17] D. J. Seo, J. Korean Phys. Soc. 45, 1575 (2004).

[18] B. J. Jin, H. S. Woo, S. Im, S. H. Bae, S. Y. Lee, Appl. Surf. Sci. 169-170, 521 (2001).

[19] T. K. Ssubramanyam, S. Uthanna, B. Srinvasulu Naidu, Mater. Lett. 35, 214 (1998).

[20] A. A. Dakhel, F. Z. Henari, Cryst. Res. Tech. 38, 979 (2011).

[21] B. Saha, S. Das, K. K. Chattopadhyay, Solar Energy Mater. Solar Cells 91, 1692 (2007).

[22] M. A. Grado-Caffaro, M. Grado-Caffaro, Mod. Phys. Lett. B 9, 1631 (1995).

[23] M. A. Grado-Caffaro, M. Grado-Caffaro, Act. Pass. Electronic Comp. 24, 57 (2001).

[24] M. A. Grado-Caffaro, M. Grado-Caffaro, Mod. Phys. Lett. B 18, 1255 (2004) .

[25] M. A. Grado-Caffaro, M. Grado-Caffaro, Optik 110, 395 (1999).

[26] M. A. Grado-Caffaro, M. Grado-Caffaro, Phys. Lett. A 372, 4858 (2008).

[27] M. A Grado-Caffaro, M. Grado-Caffaro, Mod. Phys. Lett. B 16, 747 (2002).

[28] M. A. Grado-Caffaro, M. Grado-Caffaro, Chem. Phys. Lett. 623, 72 (2015).

[29] S. K. Vasheghani Farahani, C. F. McConville, T. D. Veal, A. Schleife in: Oxide-based materials and devices IV (F.H. Teherani, D.C. Look, D.J. Rogers, Eds.), Proc. of SPIE 8626, 862604-1 (2013). 
[30] R. J. Mendelsberg, Y. Zhu, A. Anders, J. Phys. D: Appl. Phys. 45, 425302 (2012).

[31] Y. Zhu, R. J. Mendelsberg, J. Zhu, J. Han, A. Anders, J. Phys. D: Appl. Phys. 46, 195102 (2013).

[32] S. K. Vasheghani Farahani, T. D. Veal, P. D. C. King, J. Zúñiga-Pérez, V. MuñozSanjosé, C. F. McConville, J. Appl. Phys. 109, 073712.1 (2011).

[33] J. I. Pankove, Optical processes in semiconductors (Dover, New York, 1971).

[34] O. Stenzel, The physics of thin-film optical spectra: an introduction, p.214 (SpringerVerlag, 2005).

[35] M. Grundmann, The physics of semiconductors (Springer-Verlag, 2006).

[36] V. Ermakov, S. Kruchinin, H. Hori, A. Fujiwara, Int. J. Mod. Phys. B 21, 1827 (2007).

[37] A. Soldatov, N. Bogolyubov (Jr.), S. Kruchinin, Condens. Matter Phys. 9, 151 (2006).

[38] S. Kruchinin, H. Nagao, Int. J. Mod. Phys. B 26, 1230013 (2012).

[39] M. A. Grado-Caffaro, M. Grado-Caffaro, Phys. Lett. A 372, 4977 (2008).

[40] W. Zawadzki, Phys. Rev. B 74, 205439 (2006).

[41] M. A. Grado-Caffaro, M. Grado-Caffaro, Physica B 404, 1951 (2009).

[42] M. Apostol, Phys. Lett. A 372, 5093 (2008).

[43] T. M. Rusin, W. Zawadzki, Phys. Rev. B 76, 195439 (2007).

[44] M. A. Grado-Caffaro, M. Grado-Caffaro, Superlatt. Microstruct. 51, 533 (2012).

[45] M. A. Grado-Caffaro, M. Grado-Caffaro, Appl. Phys. A 103, 965 (2011).

[46] P. A. Radi, A. G. Brito-Madurro, J. M. Madurro, N. O. Dantas, Braz. J. Phys. 36, $412(2006)$.

[47] B. Gil, Phys. Rev. B 64, 201310 (2001).

[48] B. Gil, A. V. Kavokian, Appl. Phys. Lett. 81, 748 (2002).

\section{APPENDIX}

Coefficient of optical absorption

The coefficient of optical absorption can be expressed as follows:

$$
\alpha(\omega)=\frac{1}{t} \ln \frac{[1-R(\omega)]^{2}}{T(\omega)}
$$

In our case, we have that $R(\omega) \approx 0$ so, for a given photon energy, (A1) becomes:

$$
T \approx \exp -\alpha t
$$

If $\alpha t<<1$, (A2) may be approximated with its first-order McLaurin expansion yielding $T \approx 1-\alpha t$, which agrees with formula (1). 